\title{
Emprendimiento y el rol de la mujer. Caso ecuatoriano.
}

Margarita Palma S., Flor Garcés, Gloria Valencia, Wendy Wasbrum.

Recibido: enero 2017 Aprobado: mayo 2017 


\title{
Emprendimiento y el rol de la mujer. Caso ecuatoriano \\ Entrepreneurship and the role of women. Ecuadorian case
}

\author{
Margarita Palma Samaniego, Flor Garcés, Gloria Valencia Vivas, Wendy Wasbrum \\ Universidad de las Fuerzas Armadas-ESPE, Departamento de Seguridad y Defensa, Unidad Académica Especial \\ Salinas (ESMA - ESSUNA), Av. General Rumiñahui s/n, Sangolquí-Ecuador. P.O. BOX: 171-5-231B. \\ Correo: mdpalma3@espe.edu.ec, fegarces@espe.edu.ec, gmvalencia@espe.edu.ec, wewasbrun@espe.edu.ec
}

\begin{abstract}
Resumen
La ubicación de las mujeres dentro de mercados de producción y laboral ha presentado limitaciones serias, marcadas por la discriminación y estereotipos, interfiriendo en la inserción laboral de las mismas, siendo su mano de obra menos valorada que la de los hombres; evidenciándose la necesidad de mejorar su inserción y generar nuevas fuentes de trabajo; se han propuesto unidades de negocios impulsando la generación de emprendimientos que permitan incrementar de manera paulatina los ingresos del núcleo familiar. El objetivo del presente trabajo es mostrar el término emprendimiento desde el punto de vista conceptual, entendiéndose que por emprendimiento se derivan algunas teorías que permiten observar que es una característica que permite a las personas salir adelante con ideas novedosas, metodológicamente se realiza un análisis de las principales características que tienen los emprendedores observando que la edad promedio de una persona que se inicia de manera temprana en el emprendimiento es de 36 años, a pesar que a lo largo de los años ha existido una paridad al momento de emprender entre los hombres y mujeres, se observar el emprendimiento sucede con mayor proporción en las mujeres $51,1 \%$. El emprendimiento femenino se está volviendo protagonista de la economía nacional. El Global Entrepreneurship Monitor (GEM) reporta que, en Ecuador, aproximadamente, el 54\% de ellas piensan en ser microempresarias en los próximos tres años.
\end{abstract}

Palabras claves: Emprendimiento, Desarrollo económico y social, Mujer

\begin{abstract}
The location of women within the production and labor markets has presented serious limitations, marked by discrimination and stereotypes, interfering with the labor insertion of women being less valued than men. It has evidence the need to improve the insertion of women and generate new sources of work, business units have been proposed, promoting the generation of enterprises that allow the gradual increase of family income. The objective of this research is to show the term entrepreneurship from the conceptual point of view, understanding that by undertaking some theories that allow us to observe that is a feature that allows people to move forward with innovative ideas are derived methodologically an analysis of the performed main features are entrepreneurs noting that the average age of a person who starts early in the venture is 36 years, although over the years has been a parity when undertaking between men and women, will observe the venture is with higher proportion in females $51.1 \%$. Female entrepreneurship is becoming protagonist of the national economy. The Global Entrepreneurship Monitor (GEM) reports that in Ecuador, approximately 54\% of them think of being microentrepreneurs in the next three years.
\end{abstract}

Keywords: Entrepreneurship, Economic and Social Development, Women 


\section{Introducción}

Según indica (Radcliffe, 2014), en la historia de la humanidad se muestran a valiosos hombres que fueron actores de grandes logros en los diferentes espacios políticos, científicos, tecnológicos y culturales. Lo que no sucede con la acción de la mujer que aparece escasamente en la historia. Esto provoca que se genere una invisibilidad por años y desconozcamos lo que aportaron muchas mujeres al desarrollo de los pueblos, desconocimiento que se debe sin duda a que tuvieron que luchar con todas sus fuerzas en contra de los esquemas impuestos por la sociedad de su tiempo y de una absurda discriminación de género.

Según (Gallardo \& Nopo, 2009) la ubicación de las mujeres dentro de los mercados de producción y trabajo es marcada por desventajas notorias, ya que su trabajo es menos valorado que el de los hombres en relación a su calidad de trabajo y tipo de trabajo asignado.

Considerando además que, según la OIT, en América Latina la tasa desempleo urbano bajó a $6,3 \%$ y se mantendría igual a finales del 2014, siendo necesario el desafío de mejorar la calidad de los empleos. De aquí nace la necesidad de generar empleo mediante emprendimientos.

En la actualidad, existen avances importantes donde se reconoce que se debe prestar particular atención a las necesidades de las mujeres, sus diferentes roles y condiciones económicas y familiares, de tal modo que se logre intensificar su contribución como agentes efectivos en el desarrollo del capital humano, lo que significa que se debe reconocer su potencial como individuo capaz de tomar decisiones, producir y generar ingresos.

En Ecuador, reconociendo la necesidad de crear condiciones que potencien el papel de la mujer en la sociedad, incorpora formalmente en la Constitución y en el Plan Nacional del Buen Vivir, los derechos desde un enfoque de género, guardando coherencia con los acuerdos de la Plataforma de Acción de Beijing que se dio en 1995 como resultado de la IV Conferencia Mundial sobre la Mujer promovida por la Organización de las Naciones Unidas.

Considerando la necesidad de fomentar la participación de las mujeres en la economía, se propone por parte del Ministerio de Inclusión Económica y Social la implementación de programas que buscan fomentar el desarrollo social, humano y productivo de las personas que reciben el Bono de Desarrollo Humano y sus familias, a través de la generación de emprendimientos que permitan incrementar de manera paulatina los ingresos del núcleo familiar, constituyéndose en oportunidades de mejoramiento sustentable de sus condiciones de vida. Como resultado de estos programas en el año
2013, "cerca de 100 mil hogares se graduaron del Bono de Desarrollo Humano porque incursionaron en emprendimientos productivos que los sacaron de la pobreza" (Carrion, 2014).

El objetivo del presente trabajo determina los factores que influyen en el protagonismo de las mujeres en sus iniciativas emprendedoras, de manera que su aporte al desarrollo económico y social se vuelva visible. También se analiza el concepto Emprendimiento desde un enfoque investigativo analizando las teorías de varios autores donde indican que el emprender es la forma de generar ideas innovadoras y permiten a las mujeres mejorar su calidad de vida. Finalmente, se realiza un análisis de varias características de los emprendedores, donde se puede observar que el emprendimiento se genera en mayor proporción en las mujeres, y estas lo hacen por necesidad.

\section{Materiales y métodos}

El marco teórico en esta investigación está sustentado en un enfoque sistémico, partiendo de un análisis de las fuentes bibliográficas propias y particulares afín a nuestros intereses investigativos, que fundamenten teóricamente las variables de estudio y permitan cumplir con los objetivos planteados.

El presente trabajo es producto de la recopilación literaria bajo un enfoque cualitativo de diferentes autores, de forma descriptiva y exploratoria, cuyo objetivo se centra en la revisión del concepto del Emprendimiento fundamentado en fuentes como diarios, revistas o journals, libros y artículos que están disponibles en las bases de datos científicas tales como Springer-Link, Ebrary y ProQuest entre otras revistas indexadas, esto con la finalidad de obtener una caracterización y definición del concepto emprendedor.

El trabajo se desarrolla en la primera sección haciendo referencia al emprendimiento desde un enfoque investigativo, haciendo un análisis de la definición utilizando bibliografía; luego se hace un análisis de la participación de la mujer en los emprendimientos y finalmente se detallan las conclusiones.

\section{Resultados}

\section{Emprendimiento}

Al hablar de emprendimiento no se habla de un concepto nuevo, sin embargo, a lo largo de la historia 
siempre ha estado presente, por esta razón adopta varias definiciones, entre las que se puede mencionar las siguientes:

- Según (Schumpeter, 1934) describe a los emprendedores como personas innovadoras, dinámicas, fuera de lo común, soñadoras, visionarias, que actúan como destructores del equilibrio de mercado, iniciadores del cambio y creadores de nuevas oportunidades.

"La función de los emprendedores es reformar o revolucionar el patrón de la producción al explotar una inversión, o más comúnmente, una posibilidad técnica no probada. Hacerse cargo de estas cosas nuevas es difícil y constituye una función económica distinta, primero, porque se encuentran fuera de las actividades rutinarias que todos entienden, y en segundo lugar, porque el entorno se resiste de muchas maneras desde un simple rechazo a financiar o comprar una idea nueva, hasta el ataque físico al hombre que intenta producirlo" (Schumpeter, 1934).

- Según (Hoselitz, 1960), da a conocer a Jean Baptiste Say (1776 - 1832), economista francés, donde describe al emprendedor como el empresario que representa o se constituye en el catalizador para el desarrollo de productos, y lo definía como un "trabajador superior".

- Según (Jaramillo, 2008) el emprendimiento es una capacidad de los seres humanos para salir delante de manera novedosa y con ideas renovadas. $\mathrm{La}$ palabra "Emprendimiento" proviene del francés "enterpreneur" que significa pionero y se refiere a la capacidad de una persona que hace un esfuerzo adicional por alcanzar una meta u objetivo. También es utilizada para definir a la persona que inicia una nueva empresa o proyecto. Luego es asociada a los empresarios que fueron nominados como innovadores o personas que agregan valor a un producto ya existente. (Jaramillo, 2008).

- Según (Gómez, 2016), describe que la teoría del triángulo invertido propuesto por Andy Freire, indica que todo proceso de emprendimiento combinas tres elementos: Emprendedor, Idea y Capital. Esto indica que el principal apoyo del emprendedor se basa en dos elementos adicionales para llegar: la idea de negocio con viabilidad del mercado y el capital. Cuando un emprendimiento no logra el éxito deseado, se debe considerar uno de los tres elementos, así como la combinación entre ellas. (Gómez, 2016) Ver Figura 1.

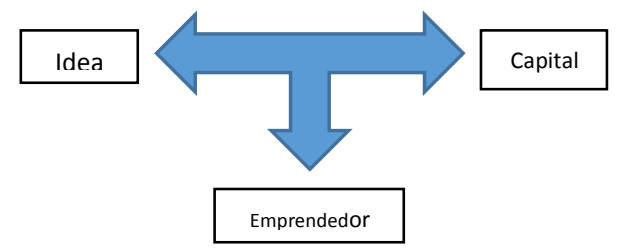

Figura 1: Elementos de Teoría Freire

Fuente: (Gómez, 2016)

Elaborado por: Autores

- Según (Kirzner, 1973), una persona emprendedora siempre está alerta de las oportunidades que se generan en situaciones de desequilibrio, esto permite que el emprendedor pueda percibir las oportunidades antes que los demás.

- Según (Grisales, 2016) el emprendimiento es algo innato en la humanidad, algo que siempre ha estado presente en el hombre, aunque claro está, el emprendimiento no se ha desarrollado en todos los hombres

Quizás el emprendimiento ha sido la diferencia entre el hombre y los demás seres vivos, pues éstos últimos, prácticamente no se han superado en miles de años, contrario al sorprendente progreso de la humanidad, y todo gracias al espíritu emprendedor que lo caracteriza.

Finalmente, haciendo una compilación de varias definiciones, se puede precisar al Emprendimiento como la actitud y aptitud de la persona que le permite emprender nuevos retos, nuevos proyectos, es lo que permite avanzar más allá de donde ha llegado. Una persona emprendedora es capaz de aprovechar las situaciones de insatisfacción, de poco crecimiento personal y laboral para desencadenar situaciones de satisfacciones, nuevos logros.

\section{Elementos que facilitan el emprendimiento}

Existen elementos que facilitan el emprendimiento, de los cuales el $67 \%$ de las menciones se concentran en 7 factores: apoyo financiero, capacidad para emprender, programas de gobierno, políticas gubernamentales, educación y entrenamiento, apertura del mercado, y normas sociales y culturales. Ver Figura 2.

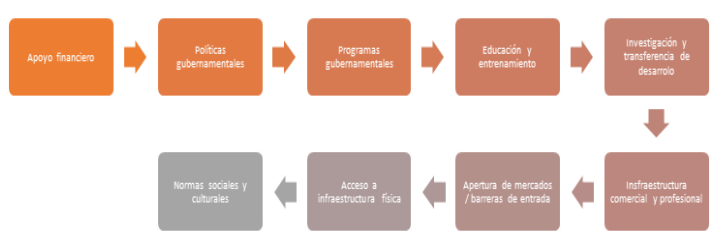

Figura 2: Elementos que facilitan el emprendimiento. Fuente: (ESPAE, Graduate School of Management, 2015) 


\section{Actividad Emprendedora}

Según el Global Entrepreneurship Monitor (GEM), el inicio de las actividades en emprendimiento se mide por el TEA (Actividad emprendedora temprana, por sus siglas en ingles), en Ecuador se mantiene una TEA alta, 33,6\% ubicándose en el primer lugar entre los países de América latina y Caribe. (ESPAE, Graduate School of Management, 2015)

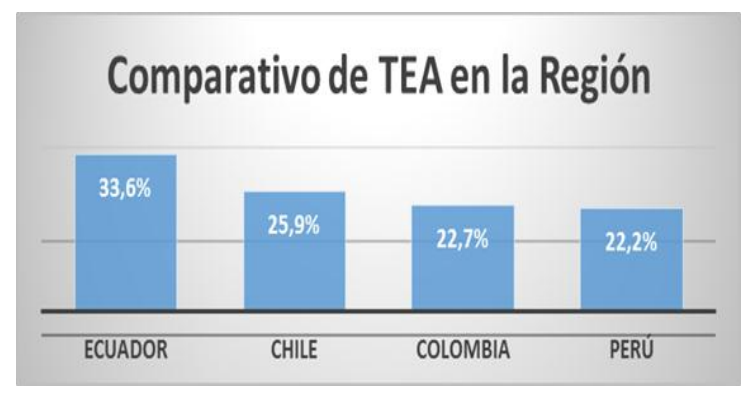

Figura 3: Comparativo del TEA en la región

Fuente: (ESPAE, Graduate School of Management, 2015)

De acuerdo al informe del GEM 2015 (ESPAE, Graduate School of Management, 2015) en orden de magnitud de la TEA, luego de Ecuador que lidera en la región, se encuentra Chile con $25.9 \%$, Colombia con $22.7 \%$, y Perú con $22.2 \%$. Chile y Perú muestran reducción en la actividad emprendedora en relación al 2014, mientras que ésta ha aumentado con respecto al año pasado para Colombia y Ecuador. Ver figura 3.

Las motivaciones en los emprendedores son la oportunidad y necesidad. Según Global Entrepreneurship Monitor 2015 (ESPAE, Graduate School of Management, 2015) . Este año el 69\% de la TEA corresponde a emprendimientos por oportunidad y el $31 \%$ por necesidad. La TEA urbana por oportunidad se reduce un poco y se observa en cambio un aumento de esta motivación para la TEA rural, manteniéndose la TEA rural por necesidad, como se observa en la Figura 4.

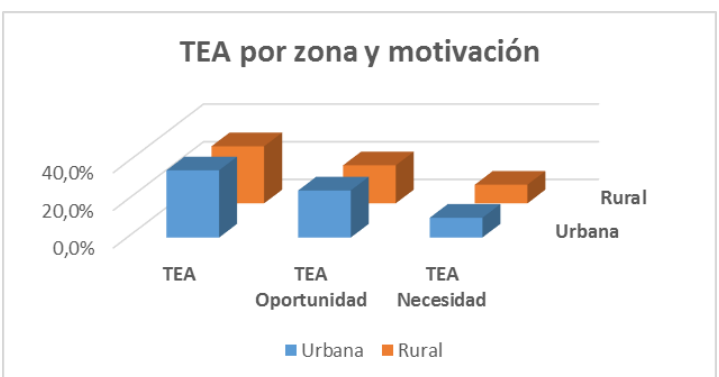

Figura 4: TEA por zona y motivación

Fuente: (ESPAE, Graduate School of Management, 2015)
Según GEM 2015 (ESPAE, Graduate School of Management, 2015) en la región, entre los países con TEA más alta, Perú muestra la mayor proporción en emprendimientos por oportunidad y Chile la menor proporción de emprendimientos por necesidad. Las motivaciones de los emprendedores de Ecuador se ubican alrededor del promedio para América Latina y el Caribe.

Del mismo modo en relación a los países con economías de eficiencia en motivación por oportunidad; en cuanto a motivación por necesidad Ecuador está por encima del promedio para economías en su misma fase de desarrollo.

El índice motivacional, es decir, la relación que existe entre los emprendedores motivados por la oportunidad de mejora y los motivados por necesidad, Ecuador se ubicó en 2015 en 1.1, similar al del 2014 y por debajo del promedio regional y de las economías de eficiencia. Al igual que en 2014, en Ecuador se establece un negocio por oportunidad de mejora por cada negocio iniciado por necesidad. (ESPAE, Graduate School of Management, 2015)

\section{Características de emprendedores}

Según (ESPAE, Graduate School of Management, 2015) en su informe GEM 2015 se muestran las principales características de los emprendedores donde se contrasta entre los que no han iniciado negocios y quienes son propietarios de negocios establecidos. Se realiza un análisis considerando características tales como Edad, Años de escolaridad, Zona de residencia, Sexo, categoría de ocupación, categoría de ingresos, oportunidades percibidas, capacidades percibidas, miedo al fracaso, intención de emprender, cierre de negocio, emprendimiento hace 2 años.

Según (ESPAE, Graduate School of Management, 2015) el informe indica que la edad promedio de emprendedores es de 36 años, tienen 10 años de escolaridad, se evidencia que la mayor parte de emprendimientos se concentra en la zona urbana, esta proporción continúa declinando. Existe mayor porcentaje de emprendimientos en las mujeres $(51,1 \%)$, el $99.3 \%$ de los emprendedores tempranos saben leer, con respecto a la ocupación de los emprendedores el $45,1 \%$ es auto empleado, $15,8 \%$ dedicado al hogar. Respecto a los ingresos mensuales el $50,4 \%$ de los emprendedores son personas cuyos ingresos son menores de $\$ 450$.

\section{Participación de la Mujer en Emprendimientos}

Según (ESPAE, Graduate School of Management, 2015) en su informe GEM 2015, expresa que hay mayor proporción de mujeres en emprendimientos, esto nos permite pensar que la mujer en las últimas 
décadas dejó de ser invisible para la economía en el Ecuador. Los aportes realizados por la mujer abarcan varias áreas como la económica, política, social y cultural. Actualmente la Constitución de Ecuador ofrece la posibilidad de un nuevo contrato social, partiendo de los derechos humanos y la calidad de la convivencia ética que existe entre hombres y mujeres.

Durante muchos años se ha observado una paridad a la hora de emprender entre hombres y mujeres. Según (ESPAE, Graduate School of Management, 2015) existen diferencias en las motivaciones de mujeres a la hora de emprender puesto que la proporción de mujeres que emprender por necesidad es superior a las de los hombres con igual motivación. Ver figura 5.

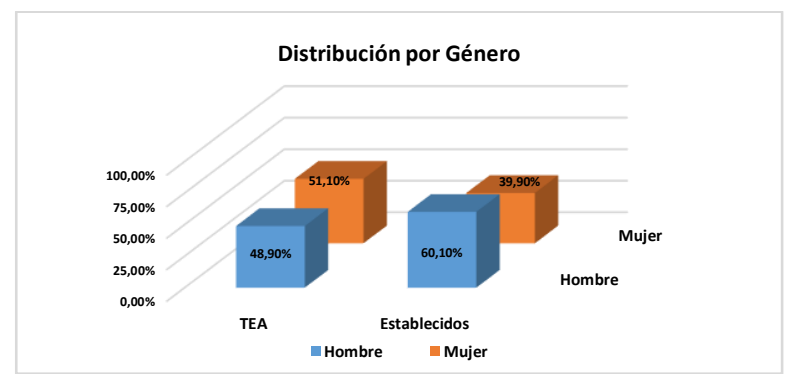

Figura 5: Distribución por Género

Fuente: (ESPAE, Graduate School of Management, 2015)

Según (Lideres, 2015), expresa que el emprendimiento femenino es motor de transformación social. Esto relacionado a que en los últimos años en Ecuador existen organismos que trabajan prioritariamente por la autonomía de las mujeres y el control de ellas sobre sus recursos mediante iniciativas productivas de la economía popular y solidaria y el acceso a créditos. En la actualidad hay que resaltar que las mujeres recurren al conocimiento en distintas áreas para poder tener participación en el desarrollo económico de los hogares, una de las formas más utilizadas es generar pequeños emprendimientos que puedan ser administrados por las mujeres y fomentar la economía.

\section{CONCLUSIONES}

Los estudios realizados muestran que Ecuador tiene muchos emprendedores, está considerado entre los 15 países con mayor tendencia a iniciar nuevos negocios. Entre las fortalezas detectadas de los emprendedores están la juventud y la capacidad de detectar oportunidades de mercados, sin embargo, las debilidades son la falta de capacitación y el capital.
Según el Monitor Global de Emprendimiento (GEM) realizado en el año 2012, evidencia que las mujeres realizan emprendimientos por sensibilidad a la necesidad, mientras que los hombres lo hacen por la oportunidad que se les presente.

El emprendimiento genera autoempleo y empleo en las localidades donde se desarrolla y el apoyo interinstitucional local y nacional en el marco del Buen vivir contribuye con la Política de Estado para que esto suceda.

\section{BIBLIOGRAFÍA}

[1] Carrión, D. S. El MIES rinde cuentas y se proyecta a futuro. MIESpacio. 2014

[2] ESPAE, Graduate School of Management. Global Entrepreneurship Monitor (GEM). Ecuador. 2015.

[3] Gallardo, L., \& Nopo, H. Ethnic and Gender wage gaps in Ecuador. InterAmerican Development Bank. 2009. [Citado 20 de mayo 2016]; 679. Disponible en:

https://publications.iadb.org/bitstream/han dle/11319/1646/Ethnic\%20and\%20Gender \%20Wage\%20Gaps\%20in\%20Ecuador.pd $\underline{\text { f? sequence }=1 \& \text { is Allowed }=\mathrm{y}}$

[4] Gómez, L. A. Teorías del Emprendimiento. 2016. [Citado 09 de septiembre de 2016]; Disponible en: http://aulavirtual.tecnologicocomfenalcovi rtual.edu.co/aulavirtual/pluginfile.php/520 365/mod_resource/content/1/TEORIAS\%2 ODEL\%20EMPRENDIMIENTO.pdf

[5] Grisales, C. P. Concepto y definición de emprendimiento. 2016. [Citado 09 de septiembre de 2016]; Disponible en: https://docs.google.com/document/d/193d A3n2hw2k3NR6hcjx9pOzvE9kYy9GNtZcLZu_Dn $\underline{\text { 8/edit }}$

[6] Hoselitz, B. F. Theories of Stages of Economic Growth. Theories of Economic Growth. 1960.

[7] Jaramillo, L. Emprendimiento: Concepto básico en competencias. Lumen. 2008. [Citado 06 de Septiembre de 2016]; Disponible

en: https://guayacan.uninorte.edu.co/divisione s/iese/lumen/ediciones/7/articulos/empren dimiento.pdf

[8] Kirzner, I.COMPETITION AND ENTREPRENEURSHIP. THE UNIVERSITY OF CHICAGO PRESS. 1973 
[9] Lideres. El emprendimiento femenino es motor de transformación. Lideres. 2015. [Citado 08 de Septiembre de 2016]; Disponible en: http://search.proquest.com/docview/16860 $54449 ?$

[10]Radcliffe, S. El género y la etnicidad como barreras para el desarrollo: Mujeres indígenas, acceso a recursos en Ecuador en perspectiva Latinoamericana. EUTOPIA: El Desarrollo Económico Territorial. 2014. 5. Disponible en:

revistas.flacsoandes.edu.ecleutopia/article /download/1486/1240
[11] Schumpeter, J. Análisis del cambio económico. (E. F. económica, Ed.) Ensayos sobre el ciclo económico. 1934 